\title{
RR Lyrae stars: New discoveries in the OGLE data
}

\author{
Radoslaw Smolec ${ }^{1}$, Henryka Netzel $^{2}$ and Igor Soszyński ${ }^{2}$ \\ ${ }^{1}$ Nicolaus Copernicus Astronomical Center \\ ul. Bartycka 18, 00-716 Warszawa, Poland \\ email: smolec@camk.edu.pl \\ ${ }^{2}$ Warsaw University Observatory \\ Al. Ujazdowskie 4, 00-478 Warszawa, Poland
}

\begin{abstract}
We report our recent analyses of the RR Lyrae Optical Gravitational Lensing Experiment (OGLE) photometry, which resulted in several new discoveries and important results. We present the in-depth analysis of the Blazhko effect discovered in several double-mode RR Lyr (RRd) stars for the first time. Typically, these stars show multiple modulation periods and somewhat atypical first overtone to fundamental mode period ratio. Our analysis of the Galactic bulge stars allowed a significant ( f factor of 10) increase of the members of the recently detected group of double-mode radial-non-radial RR Lyr stars, pulsating in the first overtone and in an yet unidentified shorter period mode, with characteristic period ratio of the two modes around 0.61. With more than 200 new members of the group first statistical analysis of the group properties is possible. Finally, we report the discovery of an even more intriguing group of double-periodic RR Lyr stars. The dominant pulsation mode in these stars corresponds to the radial first overtone. The additional period is longer, the period ratios (first overtone to a period of an yet unidentified origin) tightly cluster around 0.686 . Hence the additional period is longer than the unseen fundamental mode and cannot correspond to a purely acoustic pulsation mode.
\end{abstract}

Keywords. horizontal branch stars, stellar oscillations, RR Lyrae stars

\section{Introduction}

RR Lyrae stars are low-mass, horizontal branch stars, pulsating mostly in the radial modes: in radial fundamental mode (RRab stars), in radial first overtone mode (RRc stars) or in these two modes simultaneously (RRd stars). Non-radial modes are also detected in RR Lyr stars, mostly in RRc pulsators, but until very recently, only a dozen or so firm examples were known. A significant fraction of RR Lyr stars shows the Blazhko effect, a quasi-periodic modulation of pulsation amplitude and/or phase, origin of which remains a mystery (see e.g. Szabó 2014).

In recent years a few tens of RR Lyr stars were observed with unprecedented precision by space missions, CoRoT and Kepler (see e.g. Szabó et al. 2014, Benkö et al. 2014). Several exciting results were reported, of which the most interesting are detection of period doubling in a significant fraction of Blazhko variables (Kolenberg et al. 2010, Szabó et al. 2010) and in-depth study of the modulation properties of these stars (e.g. Guggenberger et al. 2012, Benkö et al. 2014). Other interesting result is identification of a new group of double-mode radial pulsators, pulsating simultaneously in the fundamental and in second overtone modes - for a review see Moskalik (2013). The majority of these stars were discovered thanks to space observations (Benkö et al. 2014).

The space missions, CoRoT, Kepler, and now its continuation, K2, allow very precise and high duty cycle observations, but for a very limited sample of RR Lyr stars and for a 


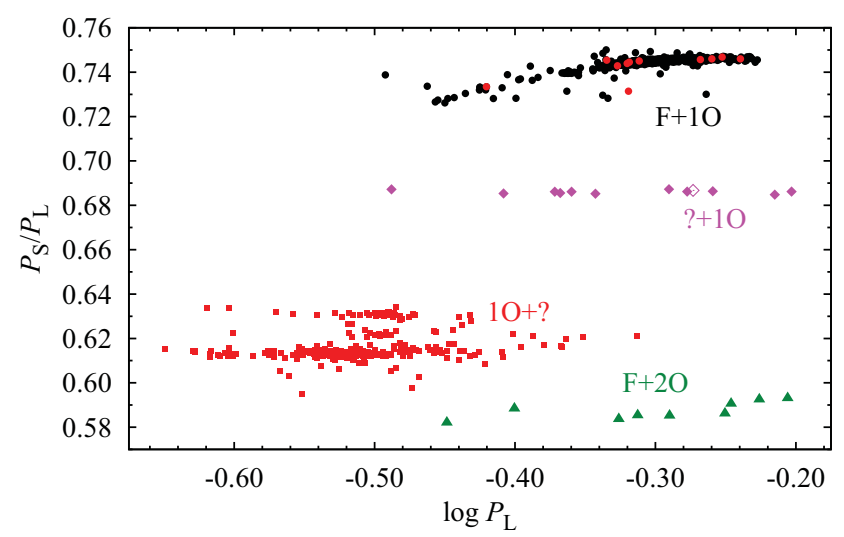

Figure 1. Petersen diagram, i.e. shorter-to-longer period ratio, $P_{\mathrm{S}} / P_{\mathrm{L}}$, versus the logarithm of the longer period, for RR Lyr stars.

limited duration (the 4-yr Kepler monitoring of the Cygnus field is an exception). In this respect, ground-based surveys are hard to beat, as they monitor significant fraction of the sky for many years. Here we focus on the Optical Gravitational Lensing Experiment (OGLE), the largest and the most successful photometric sky survey. Since the beginning of the project in 1992, OGLE was successively increasing its observing capabilities, and now, within the fourth phase of the experiment, regularly monitors the brightness of over billion stars in several hundred fields in the Galactic bulge and disc, both Magellanic Clouds with their haloes and the Magellanic bridge (Udalski et al. 2015). Only in the Galactic bulge, the OGLE-IV collection counts more than 38000 RR Lyr stars (Soszyński et al. 2014b). Such database is perfect, not only for statistical studies, but also to search for rare phenomena and peculiar objects. An RR Lyr-related examples include the discovery of binary evolution pulsator, a low mass $\left(0.26 \mathrm{M}_{\odot}\right) \mathrm{RR}$ Lyr impostor, likely a first member of a new class of variable stars (Pietrzyński et al. 2012, Smolec et al. 2013), or a few mode switching stars reported in Soszyński et al. (2014a,b). Further interesting discoveries in the OGLE data are summarised in the following Sections.

\section{Results}

New results to be reported in the following concern double-periodic or multiperiodic pulsators. The basic diagnostic tool for such stars is the Petersen diagram, in which period ratio is plotted versus the longer period - Fig. 1. In this diagram RRd stars (filled circles) follow a well defined sequence, with period ratios systematically higher for longer periods. Stars pulsating simultaneously in the fundamental and in the second overtone modes are marked with filled triangles and are centered at $P_{2} / P_{0} \approx 0.59$. Two other groups will be discussed in Sect. 2.2 and 2.3.

\subsection{Blazhko Effect in RRd stars}

So far, Blazhko effect was observed in single-mode stars, either of RRab type or of RRc type. In some modulated RRab stars second radial overtone is also detected but its amplitude is tiny as compared to fundamental mode (Benkö et al. 2014). Soszyński et al. (2014b) were the first to report the Blazhko effect in RRd stars of the OGLE Galactic bulge collection. Shortly after, Jurcsik et al. (2014) reported the modulated RRd stars in the globular cluster M3. The in-depth study of modulation in the Galactic bulge stars was conducted by Smolec et al. (2015a), and we summarise their results here. In the Petersen 


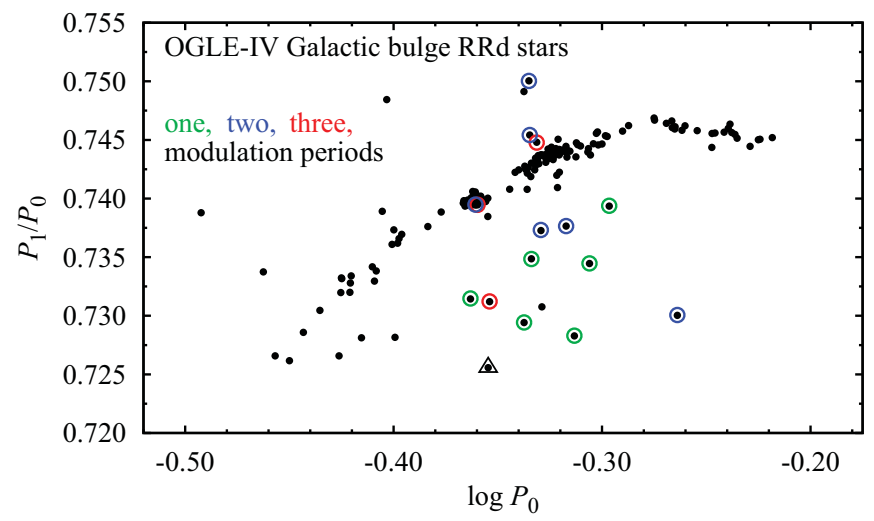

Figure 2. Petersen diagram for RRd stars of the OGLE Galactic bulge collection. Modulated RRd stars are marked with open circles. A triple-mode star with period doubling, discussed in Sect. 2.4, is marked with triangle.

diagram in Fig. 2 these stars are marked with open circles. It is striking, that nearly all these stars have a somewhat atypical period ratio, either too small, or too large as compared with the majority of RRd stars of similar fundamental mode period. Fifteen modulated RRd stars were discovered in the Galactic bulge, of which 9 are modulated with more than one period. The modulation properties of all stars are quite similar and are illustrated with OGLE-BLG-RRLYR-07393 in Fig. 3. In the left panels we show the amplitude and phase (period) variation of the two radial modes. The middle panels show the frequency spectrum of these data. Both modes are clearly modulated, but the dominant modulation periods are different; fundamental mode is modulated with a period of $P_{\mathrm{B} 2}=$ $322.9 \mathrm{~d}$, while the dominant modulation period for the first overtone is $P_{\mathrm{B} 1}=209.8 \mathrm{~d}$. The phased modulation curves are displayed in the right-most panels of Fig. 3. The larger scatter is apparent for the first overtone, because this mode is also strongly modulated with $P_{\mathrm{B} 2}$. Fundamental mode is also modulated with $P_{\mathrm{B} 1}$, very weakly, however.

In some stars we detected modulation of only one mode. In other stars, the two radial modes are modulated, but with different periods. All stars share a common feature: their modulation properties are non-stationary. Thanks to the long baseline of the OGLE photometry, we could analyse the pulsation properties of some stars over the last $14 \mathrm{yrs}$. We found that pulsation amplitudes and phases of the modulated stars are also nonstationary on a long time-scale of a few hundred to thousand days. An extreme case is OGLE-BLG-RRLYR-13442 which switched the pulsation mode, from RRab to RRd (Soszyński et al. 2014b). Fundamental mode was modulated before and after the mode switching.

\subsection{Excitation of non-radial modes: 0.61 stars}

One of the most exciting recent discoveries concerning RR Lyr stars, is detection of non-radial modes in stars with first overtone excited, i.e. in RRc or in RRd stars. The additional mode has period shorter than first overtone, the period ratios fall in a range $\sim(0.60,0.64)$. As for the majority of the stars, period ratio clusters around 0.61 (Fig. 1 ), we refer to these stars as 0.61 stars, and to the additional mode as 0.61 mode. Until recently, only 23 such stars were known (see e.g. Szabó et al. 2014, Molnár et al. 2015, Moskalik et al. 2015 and references therein), of which 13 were observed by space telescopes. This is because amplitude of the additional mode is small, typically around 2 per cent of the first overtone amplitude. Interestingly, in only one RRc star out of 14 

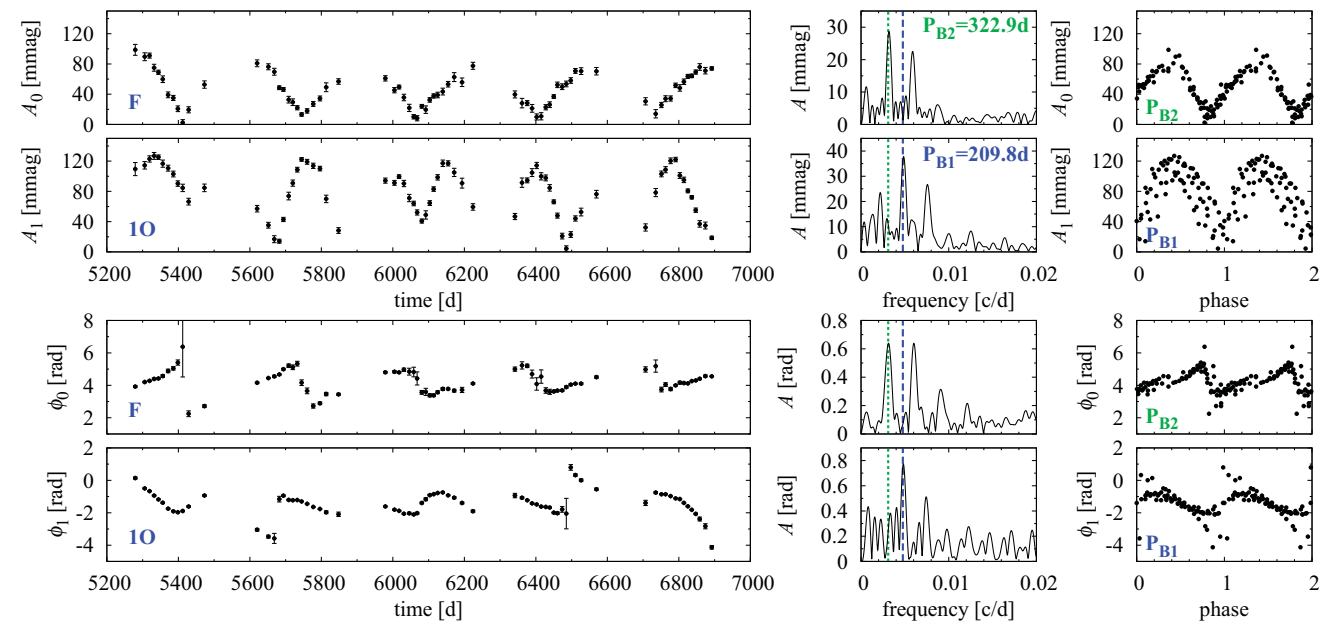

Figure 3. Variation of amplitude and phase of the radial modes of OGLE-BLG-RRLYR-07393 with time. Amplitudes/phases were extracted with the time-dependent Fourier analysis.

RRc/RRd observed from space, the additional mode was not detected. The excitation of additional 0.61 mode must be a common phenomenon. As analysed in Moskalik et al. (2015), the additional mode cannot correspond to radial mode, it must be non-radial.

Other interesting feature of these stars is the presence of sub-harmonics of the additional modes in the frequency spectrum, both at $1 / 2 f_{\mathrm{x}}$ and at $3 / 2 f_{\mathrm{x}}$. A detailed analysis of the 4 RRc stars observed by Kepler shows that the amplitude and phase associated with the additional mode and its sub-harmonics vary irregularly on a time-scales of tens of days (see figs. 6 and 7 in Moskalik et al. 2015).

The likely common excitation of non-radial modes in first overtone RR Lyr stars motivated a search for these objects in the OGLE photometry. Netzel, Smolec \& Moskalik (2015a) analysed the OGLE-III Galactic bulge photometry of RRc/RRd pulsators and reported the discovery of 147 new 0.61 stars, which constitutes only 3 per cent of the analysed sample. The small detection rate results from relatively large noise in the ground-based observations and small amplitude of the additional mode. The noise may be reduced with more frequent observations. Indeed, in their densely sampled, dedicated observations of M3, Jurcsik et al. (2015) found 18 stars with 0.61 mode, i.e. 38 per cent of their RRc/RRd sample. Analysis of the two high-cadence fields of the recently released OGLE-IV collection (Soszyński et al. 2014), led to similar result. Netzel, Smolec \& Moskalik (2015b) found the additional mode in 131 stars (of which 115 were new detections), i.e. in 27 per cent of the analysed sample. Altogether we know 304 stars of this type, of which 263 were detected in the OGLE photometry.

The OGLE stars significantly increased our knowledge about this still mysterious group. In Fig. 4 we show the Petersen diagram for the 0.61 stars only. Three sequences are well visible. The presence of the top sequence was first revealed in OGLE-III observations, while the middle, least populated sequence, became evident only after the analysis of OGLE-IV data. In 20 stars Netzel et al. (2015b) found two secondary modes belonging to two different sequences in the Petersen diagram, and in 6 stars three secondary modes, representing the three sequences, were detected. In 20 per cent of the OGLE-IV stars a signal at around sub-harmonic frequency was detected. The additional modes and their sub-harmonics are clearly non-stationary. Their amplitudes and phases vary on a time-scale of a few tens to hundred days. As a result complex patterns appear in the frequency spectra at the frequency of the additional mode and its sub-harmonics. 


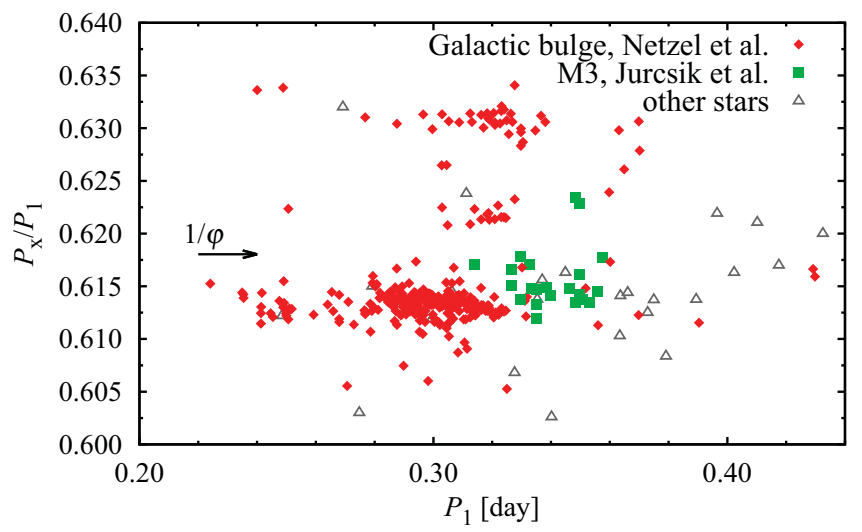

Figure 4. Petersen diagram for RR Lyr stars with the 0.61 mode. Arrow marks the reciprocal of the golden ratio.

Similar period ratios appear in first overtone Cepheids (see e.g. fig. 15 in Moskalik et al. 2015). These stars also form three sequences in the Petersen diagram. Likely, the same mechanism underlies the variability of these intriguing stars, but at the moment this mechanism is unknown (see however Dziembowski 2012). Identification of the non-radial modes is missing and the mode selection mechanism is unknown. Recently, Lindner et al. (2015) noticed that the period ratio of some of the 0.61 stars is close to the reciprocal of the golden ratio, $1 / \varphi \approx 0.618$. They argue that the dynamics driven by two frequencies in the golden ratio, maximally resist perturbations. Hence, they call these stars 'golden'. Although the idea is interesting, and the name is indeed very attractive, we refrain from calling 0.61 stars the 'golden stars', unless the connection between the golden ratio, and the phenomenon we observe, is proven. We note that in none of the stars the period ratio is equal to $1 / \varphi$ - see Fig. 4 . In fact the stars avoid the golden ratio, it falls in between the lower and middle sequences. The presence of three sequences in the Petersen diagram, and up to three non-radial modes in a single star, is an obvious difficulty for the goldenratio scenario. The presence of incommensurate frequencies, forming an irrational ratio may lead to interesting dynamical behaviour in some stars, as pointed by Lindner et al. (2015), but the connection to golden ratio seems unjustified.

\subsection{Excitation of non-radial modes: gravity modes in RR Lyr stars}

The analysis of RRc stars from the two high-cadence OGLE-IV Galactic bulge fields led to another exciting discovery - detection of a new group of double-periodic variables reported by Netzel, Smolec \& Dziembowski $(2015)$. In 11 stars ( $\sim 2$ per cent of the analysed sample), in which the dominant pulsation mode is radial first overtone, additional variability of longer period was detected. These stars are marked with diamonds in Fig. 1. The period ratios tightly cluster around $P_{1} / P_{\mathrm{x}} \approx 0.686$. A comparison with $\mathrm{RRd}$ stars leads to surprising conclusion: additional variability has period longer than period of the fundamental mode (not detected in these stars). As additional periods are too short to be associated with binarity or with rotational effects, the only plausible explanation is that additional variability corresponds to pulsation mode. As its period is longer than fundamental mode period, it must be a non-radial mode of gravity character. A literature search revealed an additional star of this type, KIC9453114, analysed by Moskalik et al. (2015), which simultaneously shows the 0.61 mode (marked with open diamond in Fig. 1). In all analysed stars the additional signal is coherent. These modes represent a challenge to stellar pulsation theory. Although instability domain may extend slightly 
beyond the fundamental mode frequency, there is no obvious selection mechanism that would favour the excitation of modes at the observed period ratio to radial first overtone.

\subsection{Peculiar multi-periodic pulsation}

The OGLE collection of RR Lyr stars contains many peculiar objects, of which OGLEBLG-RRLYR-24137 is particularly intriguing. The stars was analysed in detail by Smolec et al. (2015b). It was identified in the OGLE catalog as RRd star. The fundamental and first overtone periods are: $P_{0}=0.44192 \mathrm{~d}, P_{1}=0.32065 \mathrm{~d}$, and so the period ratio, $P_{1} / P_{0}=0.7256$, is atypical - the star is marked with triangle in Fig. 2 . It does not show the modulation of pulsation. Instead, additional mode is detected, of period shorter than first overtone period, $P_{\mathrm{x}}=0.21992 \mathrm{~d}$. The period ratio, $P_{\mathrm{x}} / P_{1}=0.686$, but the star does not belong to the group discussed in the previous section, here the additional period is shorter than first overtone period. The period ratio indicates that additional mode must be non-radial. In the frequency spectrum we also identified a significant signal at $1 / 2 f_{\mathrm{x}}$ (sub-harmonic), a signature of period doubling of the additional mode (see fig. 4 in Smolec et al. $2015 \mathrm{~b}$ ). The effect may be caused by half-integer resonance with other, unidentified mode. The star is thus extremely interesting and may be used in asteroseismic study, once all pulsation modes are identified (which is not the case yet). Hopefully it is not an isolated case, but a first member of a new group of multi-mode RR Lyr stars.

\section{Acknowledgement}

This research is supported by the Polish National Science Centre through grant DEC2012/05/B/ST9/03932 and by the Polish Ministry of Science and Higher Education through the program 'Ideas Plus' award no. IdP2012 000162. RS acknowledges the financial support from IAU.

\section{References}

Benkö, J. M., et al., 2014, ApJ Suppl. Ser., 213, 31

Dziembowski, W., 2012, Acta Astron., 62, 323

Guggenberger, E., et al., 2012, MNRAS, 424, 649

Jurcsik, J., Smitola P., Hajdu G., \& Nuspl J., 2014, ApJ, 797, L3

Jurcsik, J., et al., 2015, ApJ Suppl. Ser., 219, 25

Kolenberg, K., et al., 2010, ApJ, 713, L198

Lindner, J. F., et al., 2015, Phys. Rev. Lett., 114, 054101

Molnár, L., et al., 2015, MNRAS, 452, 4283

Moskalik, P., et al., 2013, in Suárez J.C., Garrido R., Balona L.A., Christensen-Dalsgaard J., eds, Astrophysics and Space Sci. Proc. 31, poster no. 34;

Moskalik, P., et al., 2015, MNRAS, 447, 2348

Netzel, H., Smolec, R., \& Dziembowski, W. 2015, MNRAS, 451, L25

Netzel, H., Smolec, R., \& Moskalik, P. 2015a, MNRAS, 447, 1173

Netzel, H., Smolec, R., \& Moskalik, P. 2015b, MNRAS, 453, 2022

Pietrzyński, G., et al. 2012, Nature, 484, 75

Smolec, R., et al. 2013, MNRAS, 428, 3034

Smolec, R., et al. 2015a, MNRAS, 447, 3756

Smolec, R., et al. 2015b, MNRAS, 447, 3843

Soszyński, I., et al., 2014a, Acta Astron., 64, 11

Soszyński, I., et al., 2014b, Acta Astron., 64, 177

Szabó, R., 2014, IAUS, 301, 241

Szabó, R., et al. 2010, MNRAS, 409, 1244

Szabó, R., et al. 2014, A\&AA, 570, A100

Udalski, A., Szymański, M. K., \& Szymański, G., 2015, Acta Astron., 65, 1 